\title{
Very high coronary calcium score unmasks obstructive coronary artery disease in patients with normal SPECT MPI.
}

Ghadri, J R ; Pazhenkottil, A P ; Nkoulou, R N ; Goetti, R ; Buechel, R R ; Husmann, L ; Herzog, B A ; Wolfrum, M ; Wyss, C A ; Templin, C ; Kaufmann, P A

\begin{abstract}
In patients with normal SPECT MPI, a CAC $>1000$ confers a high diagnostic added value for detecting CAD. This is not solely based on unmasking balanced ischaemia due to epicardial 3-VD, as it occurred predominantly in patients with $1-\mathrm{VD}$ and 2-VD.
\end{abstract}

DOI: https://doi.org/10.1136/hrt.2010.217281

Posted at the Zurich Open Repository and Archive, University of Zurich ZORA URL: https://doi.org/10.5167/uzh-51132

Journal Article

Accepted Version

Originally published at:

Ghadri, J R; Pazhenkottil, A P; Nkoulou, R N; Goetti, R; Buechel, R R; Husmann, L; Herzog, B A; Wolfrum, M; Wyss, C A; Templin, C; Kaufmann, P A (2011). Very high coronary calcium score unmasks obstructive coronary artery disease in patients with normal SPECT MPI. Heart, 97(12):998-1003.

DOI: https://doi.org/10.1136/hrt.2010.217281 


\section{Very high coronary calcium score unmasks obstructive coronary artery disease in patients with normal SPECT MPI}

Jelena R. Ghadri ${ }^{1 *}$, Aju P. Pazhenkottil ${ }^{1 *}$, Rene N. Nkoulou ${ }^{1}$, Robert Goetti ${ }^{1}$, Ronny R. Buechel ${ }^{1}$, Lars Husmann ${ }^{1}$, Bernhard A. Herzog ${ }^{1}$, Mathias Wolfrum ${ }^{1}$, Christophe A. Wyss ${ }^{1}$, Christian Templin ${ }^{1}$, Philipp A. Kaufmann ${ }^{1,2}$

${ }^{1}$ Cardiac Imaging, University Hospital Zurich, Switzerland

${ }^{2}$ Zurich Center for Integrative Human Physiology, University of Zurich, Switzerland

Running title: Extensive CAC unmasks CAD in normal SPECT

"The first two authors contributed equally to this work

Word count: 2723 (including: text without abstract or figure legends)

\section{Corresponding author:}

Philipp A. Kaufmann, MD

Professor and Director

Cardiac Imaging

University Hospital Zurich

Ramistrasse 100, NUK C 32

$\mathrm{CH}-8091$ Zurich

Switzerland

phone: $\quad+41-44-2554196$

fax: $\quad+41-44-2554414$

e-mail: $\quad$ pak@usz.ch

Grants: The study was supported by a grant of the Swiss National Science Foundation (SNSF) and by the ZHIP (Zurich Center for Integrative Human Physiology, University of Zurich, Switzerland). 


\section{Abstract}

Objectives: To study the clinical impact of a very high coronary artery calcium score $(C A C>1000)$ in patients with no known coronary artery disease $(C A D)$ and normal single photon emission computed tomography (SPECT) myocardial perfusion imaging (MPI). The secondary aim was to evaluate whether triple vessel disease would support the notion of balanced ischemia as an underlying mechanism of false negative SPECT MPI in patients with very high CAC.

Background: No clinical data exists on the clinical value of extensive CAC in patients with normal SPECT MPI.

Methods: 50 patients with suspected CAD and normal stress/rest SPECT MPI and CAC $>1000$ prospectively underwent invasive coronary angiography as standard of reference. Coronary lesions with $\geq 50 \%$ luminal diameter narrowing on invasive coronary angiography were considered as significant stenosis.

Results: The median total CAC was 1975 (range 1018-8046). In $37 / 50$ (74\%) patients coronary angiography revealed 1-vessel disease (VD) $(n=15), 2-V D(n=10)$ or 3-VD $(n=12)$. Twenty-six revascularizations (percutaneous coronary intervention/ coronary artery bypass grafting) were performed in 7 (6/1), 7 (6/1) and 12 (7/5) patients with 1VD, 2-VD and 3-VD, respectively.

Conclusions: In patients with normal SPECT MPI a CAC $>1000$ confers a high diagnostic added value for detecting CAD. This is not solely based on unmasking balanced ischemia due to epicardial 3-VD as it occurred predominately in patients with 1-VD and 2-VD.

Key Words: Coronary artery disease, Coronary calcium score, balanced ischemia, normal SPECT/MPI 
Word count: 225 


\section{Abbreviations}

$\mathrm{CAD}=$ coronary artery disease

$\mathrm{CAC}=$ coronary artery calcium score

$\mathrm{CT}=$ computed tomography

$E C G=$ electrocardiogram

LVEF = left ventricular ejection fraction

$\mathrm{MPI}=$ myocardial perfusion imaging

$\mathrm{PET}=$ positron emission tomography

SPECT $=$ single photon emission computed tomography

SSS $=$ summed stress score

TID = transient ischemic dilation

VD = vessel disease 


\section{Introduction}

Nuclear myocardial perfusion imaging (MPI) with single photon emission computed tomography (SPECT) is a well-established non-invasive method for detecting ischemia and the prognostic evaluation of patients with known or suspected coronary artery disease (CAD). $\underline{\text { A large body of literature has provided good evidence that }}$ normal SPECT MPI scan has a very high negative predictive value for ischemic heart disease and cardiovascular events in a general population. Atherosclerosis as an early stage of coronary heart disease is frequently associated with coronary calcifications ${ }^{1-4}$ Recently, the coronary calcium (CAC) score has emerged as a new technique with incremental prognostic value compared to conventional cardiovascular risk factors. High values of CAC are often an indicator of stenotic lesions and are associated with an increased risk of cardiovascular events. ${ }^{5-8}$ Because of the large discrepancy between the anatomic extent of CAD and ischemia - since many stenoses do not result in abnormal perfusion - the combination of the morphological anatomic information from CAC with functional information obtained from MPI may provide complementary information for the clinician. ${ }^{9-13}$ Due to the widespread availability of multislice computed tomography (CT) scanners and particularly with the integration of the latter into hybrid SPECT/CT or positron emission tomography (PET)/CT devices where low dose non-contrast CT scan for attenuation correction can be used for calculating CAC the clinical experience with CAC is growing. ${ }^{8,14}$ However, in a small but important subset of patients results from the two modalities have been conflicting, i.e. normal SPECT MPI despite very high CAC. ${ }^{15-17}$ In a recent pilot study a cut off value for CAC at 709 was identified for the prediction of angiographic CAD despite normal SPECT MPI findings, suggesting balanced ischemia in multivessel disease. ${ }^{8}$ Although these data support the notion 
that CAC may offer incremental diagnostic information over SPECT data for identifying patients with significant CAD and negative MPI results, systematic data is lacking.

Therefore, the aim of the present study was to prospectively identify the rate of obstructive CAD in patients with normal SPECT MPI and a CAC $>1000$. This cut off value was chosen as previously suggested ${ }^{16,18}$ - although slightly higher than the value described above ${ }^{8}$ - in order to ensure a high specificity in detection of clinically relevant $\mathrm{CAD}$ in agreement with the position statement of the working group on Nuclear Cardiology and Cardiac CT of the European Society of Cardiology classifying a CAC >1000 as extensive. ${ }^{19}$ A secondary aim was to evaluate whether 3vessel disease (VD) would support the notion of balanced ischemia as an underlying mechanism of false negative SPECT MPI in the presence of CAC $>1000$. 


\section{Methods}

\section{Study population}

The present study included 50 consecutive patients with a suspected but no known $\underline{\text { CAD normal gated-SPECT and a CAC }>1000 \text {. A gated-SPECT result was considered }}$ as normal as follows: no visual perfusion defect, summed stress score (SSS) $<3$, a left ventricular ejection fraction (LVEF) at rest (female/male) $>50 / 45 \%{ }^{20}$ and regular heart rate. An invasive coronary angiography served as a standard of reference and was performed based on previous data indicating in patients with normal SPECT MPI and high CAC a high incidence of angiographic $C A D^{8}$ and an adverse outcome. ${ }^{16-17}$ Despite lack of ischemia in SPECT a further diagnostic evaluation is also supported by studies reporting that high CAC is associated with extremely elevated risk of cardiac hard events. Written informed consent was obtained from all patients.

\section{Gated SPECT myocardial perfusion imaging}

Gated SPECT data acquisition was performed on a dual-head detector camera (Infinia or Ventri, GE Healthcare, Milwaukee, WI, USA), with a 1-day pharmacologic stress/rest MPI protocol using a weight-adjusted dose of 300 to $400 \mathrm{MBq}$ of ${ }^{99 \mathrm{~m}} \mathrm{TC}$ tetrofosmin for stress (adenosine at standard rate of $0.14 \mathrm{mg} / \mathrm{kg} / \mathrm{min}$ over 6 minutes) and a 3 fold higher dose of ${ }^{99 \mathrm{~m}} \mathrm{Tc}$-tetrofosmin for rest image acquisition. SPECT data was analyzed by two experienced imaging cardiologists with regard to the presence of reversible and/or fixed perfusion defects on short-axis, horizontal and vertical longaxis slices as well as on the polar maps with a commercially available quantitative gated and perfusion SPECT software package (Cedars QGS/QPS; Cedars-Sinai Medical Center) which also allowed assessment of LVEF. In addition MPIs were assessed semi-quantitatively by segmental visual interpretation. A scan was 
considered as normal if the summed stress score was $<3 .{ }^{21-22}$ The use of low isotope dose in the first part of the one day protocol precludes meaningful assessment of transient ischemic dilatation (TID). As no values of transient ischemic dilatation (TID) have been validated for low-dose ECG-gated acquisition 60 minutes after vasodilator stress, we only provided visual assessment of TID using a 0-3 visual score $(0=$ none, $1=$ equivocal, $2=$ definite but moderate, and $3=$ severe), with $\geq 2$ being considered abnormal, as previously reported..$^{23}$

\section{Coronary artery calcium score}

A low-dose, unenhanced CT for SPECT MPI attenuation correction was performed on a LightSpeed VCT XT 64-slice CT standalone scanner (GE Healthcare) with the following scanning parameters: prospective ECG-triggering, $2.5 \mathrm{~mm}$ slice thickness, $120 \mathrm{kV}$ tube voltage, $200 \mathrm{mAs}$ per rotation tube current, and a large scan field-ofview of $50 \times 50 \mathrm{~cm}$. Co-registration was accomplished by visual quality control of emission (SPECT) and transmission scans (CT) using a commercially available and well established tool intergraded in the QGS/QPS software as previously reported. ${ }^{24}$ No beta blocker was administered, and the average heart rate during acquisition was $62 \pm 5 \mathrm{bpm}$. From this scan Agatston scores were computed with commercially available software (Smartscore, GE Healthcare), as previously reported. ${ }^{24}$

\section{Coronary angiography}

Invasive coronary angiography through the femoral artery was performed on an Allura 9 and an Allura XPER FD 10/10 (Philips Medical Systems) catheterisation equipment in an experienced catheterisation laboratory of a teaching hospital (University Hospital Zurich) following a protocol, which consists of a biplane angiography of left coronary artery with two radiation exposures in four orientations 
and of the right coronary artery with two exposures in two orientations, as is routinely performed in our cardiology department as previously reported. ${ }^{25}$ Additional views could be performed at the operator's discretion if necessary.

At angiography, coronary artery lesions were considered to be significant stenoses when there was $\geq 50 \%$ luminal diameter narrowing (figure 1). CAD was defined as the presence of at least one significant stenosis. Patients with left main stenosis were categorized as 2-VD.

The decision of the operator to perform percutaneous coronary intervention $(\mathrm{PCl})$ or recommend coronary artery bypass graft (CABG) surgery was prospectively recorded. Lesions severity in coronary angiograms was visually assessed by two $\underline{\text { experienced interventional cardiologists and in case of disagreement a consensus }}$ between both was reached. The operators based this decision on their clinical judgment integrating the visual angiography finding with all available clinical information. No further complementary functional parameters such as for example the recently promoted functional flow reserve ${ }^{26}$ were available.

\section{Radiation Dose}

Values for effective radiation dose of low dose CT were calculated by multiplying the dose length product (DLP) with a conversion factor $(k=0.014 \mathrm{mSv} / \mathrm{mGy} \times \mathrm{cm})$ as previously described ${ }^{27}$ and adopted in large trials. ${ }^{28}$ For estimating the effective radiation dose from SPECT MPI, we used a conversion factor of $6.9 \times 10^{-3 \mathrm{mSV}} / \mathrm{MBq}^{29}$

\section{Statistical analysis}

Quantitative intergroup comparisons were performed with the Wilcoxon test (two groups) or the Kruskal-Wallis test (more than two groups). Variables were expressed as mean \pm standard deviation or median and range and categorical variables as 
frequencies and percentages. CAC distribution between the main epicardial vessels was compared with the Friedman test. A p-value of $<0.05$ was considered to be statistically significant. SPSS software (version 15.0, SPSS Inc.; Chicago Illinois) was used for statistical testing. 


\section{Results}

\section{Study population}

The mean age of the study population was $67 \pm 9$ years, $72 \%$ were male. The mean number of risk factors was $2.5 \pm 1.2$ and most patients were classified as intermediate risk by Framingham risk score which averaged $15.7 \pm 7.3$ (median 15.5, range 3-31). The characteristics of the study population are given in table 1.

\section{SPECT}

According to the inclusion criteria following the study design all MPI at stress and rest were normal. The mean values for the summed rest, stress, and difference scores were $0.3 \pm 0.4,0.7 \pm 0.9,0.5 \pm 0.5$. Mean LVEF was $63.7 \pm 4.3 \%$ at rest, and $62.8 \pm 6.3 \%$ after Adenosine-stress ( $p=n . s$.$) . There was no significant adenosine-induced ST$ segment depression. TID score was 0 in 49 patients and equivocal in 1 patient.

\section{Coronary angiography}

Thirty-seven (74\%) of the 50 patients had a significant CAD. This included 15 patients with 1-VD (30\%), 10 patients with 2-VD (20\%) and 12 patients with 3-VD (24\%) (figure 2A). Thus, in the majority of patients $(78 \%)$ with unmasked CAD the underlying disease was not 3-VD (figure 2B). Twenty-six of the 37 CAD patients $(70 \%)$ underwent coronary revascularization, i.e. $\mathrm{PCl}(\mathrm{n}=19)$ or CABG $(\mathrm{n}=7)$. Among 37 patients with CAD 28 patients revealed a stenosis $\geq 80 \%$. While $\mathrm{PCl}$ was evenly distributed among 1-VD, 2-VD and 3-VD patients, CABG was predominately performed in those with 3-VD (table 2). Remarkably, in 6 of the 13 patients without angiographic CAD the absence of any luminal irregularities was reported from angiography, despite massive calcification. 


\section{Coronary artery calcium score}

The median CAC of the study population was 1975 (range 1018-8046), while the respective values for patients with and without CAD was 1930 (range 1026-8046) and 1977 (range 1018-3007). The median CAC in patients with 1-VD was 1476 (range 1026-5009) and tended to be higher in 2-VD and 3-VD, i.e. 2289 (range 1294-8046) and 2190 (range 1332-5915) although the difference fell short of statistical significance $(p=0.15)$ (figure 2). Median CAC was significantly lower in LMA (52, range 0-751) and RCA (279, range 0-2884) compared to LAD (686, range $\underline{0-751)(p \leq 0.001)}$.

\section{Radiation dose from SPECT MPI and Coronary artery calcium score}

The average DLP for CAC measurement was $72.2 \pm 22.2 \mathrm{mGy} \times \mathrm{cm}$ resulting in a dose estimation of $1.0 \pm 0.3 \mathrm{mSv}$. Estimated effective dose from SPECT MPI was $\underline{8.5 \pm 1.2 \mathrm{mSv}^{29}}$ 


\section{Discussion}

The present data indicate that a CAC $>1000$ is a predictor of CAD in patients with intermediate pretest probability for CAD and normal SPECT MPI as angiographically significant lesion were found in $74 \%$ of the study population. The presence of atherosclerosis does not necessarily reflect obstructive coronary lesions and conversely coronary lesions do not necessarily confer hemodynamic relevance. However, an increasing CAC is generally associated with an increase in abnormal SPECT findings. ${ }^{10,12-13}$ For example, Anand et al. observed a prevalence of silent ischemia in $18 \%$ of patients with a CAC of $100-400$, while the prevalence increased to $45 \%$ in patients with CAC $>400 .{ }^{30}$ It has been reported that a high CAC in patients with normal SPECT MPI reflects non obstructive atherosclerosis which should be recognized as a preclinical state with strong predictive value for the development of $C A D^{5}$ and should therefore, encourage aggressive risk factor modification according to NCEP guidelines. ${ }^{31}$

Our study extends these observations towards the upper end of the CAC spectrum. In patients with massive coronary calcifications these not only reflect an increased cardiovascular risk ${ }^{5}$, but more importantly extensive coronary calcifications with a CAC >1000 appear to unmask obstructive CAD undetected by SPECT MPI. Although the pooled literature reports an overall sensitivity of SPECT MPI to detect multivessel disease ranging from $80 \%-95 \%^{32-33}$, only in $29 \%$ of patients with $3-\mathrm{VD}$ defects are found in all three territories. ${ }^{34}$ By its nature, SPECT allows only detection of relative perfusion defects. Therefore, the true extent of myocardial ischemia may be underestimated particularly in vascular territories adjacent to areas with more severe coronary stenosis. 
Consequently, in cases with evenly distributed ischemia without inducible flow heterogeneity among the different vessel territories balanced ischemia has been hypothesized to explain lack of perfusion defects. This however, is unlikely to be a main contributing factor to the results of the present study because a 3-VD was found in less than a third of the CAD patients. The concept of balanced ischemia may not be solely based on balanced epicardial coronary lesion severity, but rather could also be caused by microcirculatory dysfunction ${ }^{35-36}$ where global hyperemic myocardial flow response and myocardial flow reserve are blunted. ${ }^{31} \mathrm{~A}$ massive increase in CAC would then - although measured in the epicardial vessel - reflect involvement of the coronary microcirculatory tree, potentially associated with a decrease in myocardial flow reserve favoring a balanced hyperemic response due to blunted flow heterogeneity. This is in line with a recent study documenting that a high CAC is paralleled by endothelial dysfunction. ${ }^{37}$ There is increasing evidence that even nonobstructive CAD with coronary calcification may impair hyperemic flow increases as determined with PET or MRI. ${ }^{39-41}$ Concordantly, Pirich et al. have reported that relevant coronary calcification can be confined to one epicardial coronary vessel, while myocardial flow reserve is reduced in all vascular territories, therefore not revealing any perfusion abnormality by visual image analysis. ${ }^{38}$ This was further substantiated by the fact that flow response was significantly lower in patients with

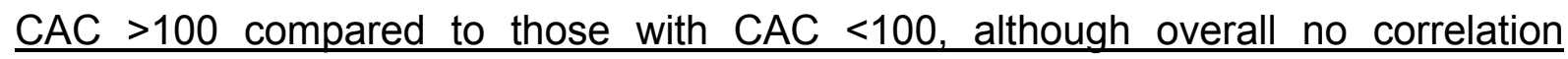
between $\mathrm{CAC}$ and flow reserve was found. This, however, may have been due to the study design including only patients with relatively low CAC (median 142, range 0 915), where the vascular reactivity is not yet strongly affected. By contrast our study population with a median CAC of 1974 (range 1018-8046) represents a more advanced stadium of CAD and atherosclerosis in which microcirculatory involvement is very likely to be present even in myocardial segments subtended by non-stenotic 
coronary arteries as previously reported. ${ }^{42}$ This may provide the ground for balanced ischemia on the microcirculatory level of the coronary tree in patients with extensive coronary calcifications without 3-VD. This is further supported by a previous study reporting obstructive lesions in $12 \%$ of territories with normal SPECT MPI but massive calcifications. ${ }^{43}$

Study limitations: It may be perceived as a limitation of the present study, that the classification of coronary lesions as significant was not complemented by a functional assessment such as fractional flow reserve. The latter was not yet implemented into our daily routine during our study which was completed before the solid data from Tonino et al. ${ }^{26}$ became available and before FFR has been adopted in the latest ESC guidelines for revascularization. ${ }^{44}$ Therefore, the standard of reference was solely based on visual evaluation of luminal narrowing. This, however, reflects common daily practice in many coronary catheterization laboratories ${ }^{45}$ and is in line with recent randomized trials evaluating coronary revascularization, like the COURAGE (Clinical Outcomes Utilizing Revascularization and Aggressive Drug Evaluation), BARI 2D (Bypass Angioplasty Revascularization Investigation 2 Diabetes), and SYNTAX (Synergy Between Percutaneous Coronary Intervention With TAXUS and Cardiac Surgery) studies, in which lesions with diameter stenosis $\geq 50 \%$ based on visual estimation of the angiogram were generally considered for revascularization. ${ }^{46-48}$ An additional limitation may be the fact that our definition of normal MPI was mainly based on normal findings of perfusion and rest LVEF as our protocol with scanning late after adenosine stress does not allow to benefit from the incremental diagnostic ${ }^{49}$ and prognostic ${ }^{23}$ value of TID or stress induced wall motion abnormalities to identify CAD. However, the prevalence of CAC $>1000$ in normal SPECT MPI approximates $7 \%$ at our institution comparing favorably to the reported range of $9-11 \% .^{10,16-17}$ This underlines the validity of our method to classify a SPECT scan as normal, because 
classifying scans inappropriately as normal would result in a higher incidence of CAC $\geq 1000$.

Furthermore, the present study was focused on the prevalence of epicardial coronary $\underline{\text { lesions in patients with normal myocardial perfusion and the diagnostic value of }}$ extensive coronary calcification to unmask CAD. Therefore, the study was not powered and not designed to provide outcome data.

Finally, whether adjustment of the cut-off value for CAC would be appropriate in $\underline{\text { different patient populations with different pre-test likelihood must remain a matter of }}$ discussion as our data do not allow commenting on this.

Conclusions: In intermediate risk patients with normal MPI a CAC >1000 confers an incremental diagnostic value for detecting CAD. This is not solely based on unmasking balanced ischemia due to epicardial 3-VD as it occurred predominantly in patients with 1-VD and 2-VD. Therefore, further evaluation of CAD with invasive coronary angiography including FFR and / or initiation of aggressive optimal medical therapy should be considered in these patients. 


\section{Acknowledgment}

We are grateful to Ennio Mueller, Edlira Loga, Sabrina Epp, Mirjam De Bloeme and Raji Kanagasabai for their excellent technical support and Patrick von Schulthess for careful image reconstruction.

\section{Conflict of interest}

None.

\section{Patient consent}

Obtained.

\section{Ethics approval}

This study was conducted with the approval of the local ethics committee of the University Hospital Zurich. 


\section{References}

1. Simons DB, Schwartz RS, Edwards WD, et al. Noninvasive definition of anatomic coronary artery disease by ultrafast computed tomographic scanning: a quantitative pathologic comparison study. J Am Coll Cardiol 1992;20:1118-26.

2. Mautner GC, Mautner SL, Froehlich J, et al. Coronary artery calcification: assessment with electron beam CT and histomorphometric correlation. Radiology 1994;192:619-23.

3. Sangiorgi G, Rumberger JA, Severson A, et al. Arterial calcification and not lumen stenosis is highly correlated with atherosclerotic plaque burden in humans: a histologic study of 723 coronary artery segments using nondecalcifying methodology. J Am Coll Cardiol 1998;31:126-33.

4. McCarthy JH, Palmer FJ. Incidence and significance of coronary artery calcification. Br Heart J 1974;36:499-506.

5. Detrano R, Guerci AD, Carr JJ, et al. Coronary calcium as a predictor of coronary events in four racial or ethnic groups. N Engl J Med 2008;358:1336-45.

6. Arad $\mathrm{Y}$, Goodman KJ, Roth $\mathrm{M}$, et al. Coronary calcification, coronary disease risk factors, C-reactive protein, and atherosclerotic cardiovascular disease events: the St. Francis Heart Study. J Am Coll Cardiol 2005;46:158-65.

7. Haberl R, Becker A, Leber A, et al. Correlation of coronary calcification and angiographically documented stenoses in patients with suspected coronary artery disease: results of 1,764 patients. J Am Coll Cardiol 2001;37:451-7.

8. Schepis $\mathrm{T}$, Gaemperli $\mathrm{O}$, Koepfli $\mathrm{P}$, et al. Added value of coronary artery calcium score as an adjunct to gated SPECT for the evaluation of coronary artery disease in an intermediate-risk population. $J$ Nucl Med 2007;48:1424-30. 
9. Gaemperli O, Schepis T, Valenta I, et al. Functionally relevant coronary artery disease: comparison of 64-section CT angiography with myocardial perfusion SPECT. Radiology 2008;248:414-23.

10. Berman DS, Wong ND, Gransar H, et al. Relationship between stress-induced myocardial ischemia and atherosclerosis measured by coronary calcium tomography. J Am Coll Cardiol 2004;44:923-30.

11. Thompson RC, McGhie Al, Moser KW, et al. Clinical utility of coronary calcium scoring after nonischemic myocardial perfusion imaging. $J$ Nucl Cardiol 2005;12:392-400.

12. Moser KW, O'Keefe JH, Jr., Bateman TM, et al. Coronary calcium screening in asymptomatic patients as a guide to risk factor modification and stress myocardial perfusion imaging. J Nucl Cardiol 2003;10:590-8.

13. He ZX, Hedrick TD, Pratt CM, et al. Severity of coronary artery calcification by electron beam computed tomography predicts silent myocardial ischemia. Circulation 2000;101:244-51.

14. Burkhard N, Herzog BA, Husmann L, et al. Coronary calcium score scans for attenuation correction of quantitative PET/CT 13N-ammonia myocardial perfusion imaging. Eur J Nucl Med Mol Imaging 2010;37:517-21.

15. Chang SM, Nabi F, Xu J, et al. The coronary artery calcium score and stress myocardial perfusion imaging provide independent and complementary prediction of cardiac risk. J Am Coll Cardiol 2009;54:1872-82.

16. Rozanski A, Gransar H, Wong ND, et al. Clinical outcomes after both coronary calcium scanning and exercise myocardial perfusion scintigraphy. J Am Coll Cardiol 2007;49:1352-61.

17. Schenker MP, Dorbala S, Hong EC, et al. Interrelation of coronary calcification, myocardial ischemia, and outcomes in patients with intermediate likelihood of 
coronary artery disease: a combined positron emission tomography/computed tomography study. Circulation 2008;117:1693-700.

18. Budoff MJ, Shaw LJ, Liu ST, et al. Long-term prognosis associated with coronary calcification: observations from a registry of 25,253 patients. J Am Coll Cardiol 2007;49:1860-70.

19. Perrone-Filardi $\mathrm{P}$, Achenbach $\mathrm{S}$, Mohlenkamp $\mathrm{S}$, et al. Cardiac computed tomography and myocardial perfusion scintigraphy for risk stratification in asymptomatic individuals without known cardiovascular disease: a position statement of the Working Group on Nuclear Cardiology and Cardiac CT of the European Society of Cardiology. Eur Heart J 2010.

20. Sharir T, Germano G, Kavanagh PB, et al. Incremental prognostic value of poststress left ventricular ejection fraction and volume by gated myocardial perfusion single photon emission computed tomography. Circulation 1999;100:1035-42.

21. Hachamovitch R, Berman DS, Shaw LJ, et al. Incremental prognostic value of myocardial perfusion single photon emission computed tomography for the prediction of cardiac death: differential stratification for risk of cardiac death and myocardial infarction. Circulation 1998;97:535-43.

22. Berman DS, Kiat H, Friedman JD, et al. Separate acquisition rest thallium201/stress technetium-99m sestamibi dual-isotope myocardial perfusion singlephoton emission computed tomography: a clinical validation study. J Am Coll Cardiol 1993;22:1455-64

23. Abidov A, Bax JJ, Hayes SW, et al. Transient ischemic dilation ratio of the left ventricle is a significant predictor of future cardiac events in patients with otherwise normal myocardial perfusion SPECT. J Am Coll Cardiol 2003;42:1818-25. 
24. Schepis T, Gaemperli O, Koepfli P, et al. Use of coronary calcium score scans from stand-alone multislice computed tomography for attenuation correction of myocardial perfusion SPECT. Eur J Nucl Med Mol Imaging 2007;34:11-9.

25. Herzog BA, Wyss CA, Husmann L, et al. First head-to-head comparison of effective radiation dose from low-dose 64-slice CT with prospective ECGtriggering versus invasive coronary angiography. Heart 2009;95:1656-61.

26. Tonino PA, De Bruyne $\mathrm{B}$, Pijls $\mathrm{NH}$, et al. Fractional flow reserve versus angiography for guiding percutaneous coronary intervention. $N$ Engl $J$ Med 2009;360:213-24.

27. Einstein AJ, Moser KW, Thompson RC, et al. Radiation dose to patients from cardiac diagnostic imaging. Circulation 2007;116:1290-305.

28. Hausleiter J, Meyer T, Hermann F, et al. Estimated radiation dose associated with cardiac CT angiography. JAMA 2009;301:500-7.

29. Icrp. Radiation dose to patients from radiopharmaceuticals. Addendum 3 to ICRP Publication 53. ICRP Publication 106. Approved by the Commission in October 2007. Ann ICRP 2008;38:1-197.

30. Anand DV, Lim E, Raval U, et al. Prevalence of silent myocardial ischemia in asymptomatic individuals with subclinical atherosclerosis detected by electron beam tomography. J Nucl Cardiol 2004;11:450-7.

31. Grundy SM, Cleeman JI, Merz CN, et al. Implications of recent clinical trials for the National Cholesterol Education Program Adult Treatment Panel III guidelines. Circulation 2004;110:227-39.

32. Mahmarian JJ, Boyce TM, Goldberg RK, et al. Quantitative exercise thallium201 single photon emission computed tomography for the enhanced diagnosis of ischemic heart disease. J Am Coll Cardiol 1990;15:318-29. 
33. DePasquale EE, Nody AC, DePuey EG, et al. Quantitative rotational thallium201 tomography for identifying and localizing coronary artery disease. Circulation 1988;77:316-27.

34. Christian TF, Miller TD, Bailey KR, et al. Noninvasive identification of severe coronary artery disease using exercise tomographic thallium-201 imaging. Am J Cardiol 1992;70:14-20.

35. Kaufmann PA, Camici PG. Myocardial blood flow measurement by PET: technical aspects and clinical applications. J Nucl Med 2005;46:75-88.

36. Camici PG, Crea F. Coronary microvascular dysfunction. N Engl J Med $2007 ; 356: 830-40$.

37. Huang PH, Chen LC, Leu HB, et al. Enhanced coronary calcification determined by electron beam $\mathrm{CT}$ is strongly related to endothelial dysfunction in patients with suspected coronary artery disease. Chest 2005;128:810-5.

38. Pirich C, Leber A, Knez A, et al. Relation of coronary vasoreactivity and coronary calcification in asymptomatic subjects with a family history of premature coronary artery disease. Eur J Nucl Med Mol Imaging 2004;31:66370.

39. Curillova Z, Yaman BF, Dorbala S, et al. Quantitative relationship between coronary calcium content and coronary flow reserve as assessed by integrated PET/CT imaging. Eur J Nucl Med Mol Imaging 2009;36:1603-10.

40. Schindler TH, Facta AD, Prior JO, et al. Structural alterations of the coronary arterial wall are associated with myocardial flow heterogeneity in type 2 diabetes mellitus. Eur J Nucl Med Mol Imaging 2009;36:219-29.

41. Wang $L$, Jerosch-Herold $M$, Jacobs DR, Jr., et al. Coronary artery calcification and myocardial perfusion in asymptomatic adults: the MESA (Multi-Ethnic Study of Atherosclerosis). J Am Coll Cardiol 2006;48:1018-26. 
42. Beanlands RS, Muzik O, Melon P, et al. Noninvasive quantification of regional myocardial flow reserve in patients with coronary atherosclerosis using nitrogen13 ammonia positron emission tomography. Determination of extent of altered vascular reactivity. J Am Coll Cardiol 1995;26:1465-75.

43. Schuijf JD, Wijns W, Jukema JW, et al. A comparative regional analysis of coronary atherosclerosis and calcium score on multislice CT versus myocardial perfusion on SPECT. J Nucl Med 2006;47:1749-55.

44. Wijns W, Kolh P, Danchin N, et al. Guidelines on myocardial revascularization: The Task Force on Myocardial Revascularization of the European Society of Cardiology (ESC) and the European Association for Cardio-Thoracic Surgery (EACTS). Eur Heart J 2010;31:2501-55.

45. Cutlip DE, Mehran R, Vranckx P. FAME and Coronary Stent Investigations - Is There a Kink in the Wire? J Am Coll Cardiol 2010;57:115-6.

46. Boden WE, O'Rourke RA, Teo KK, et al. Optimal medical therapy with or without PCI for stable coronary disease. N Engl J Med 2007;356:1503-16.

47. Frye RL, August $\mathrm{P}$, Brooks $\mathrm{MM}$, et al. A randomized trial of therapies for type 2 diabetes and coronary artery disease. N Engl J Med 2009;360:2503-15.

48. Serruys PW, Morice MC, Kappetein AP, et al. Percutaneous coronary intervention versus coronary-artery bypass grafting for severe coronary artery disease. N Engl J Med 2009;360:961-72.

49. Weiss AT, Berman DS, Lew AS, et al. Transient ischemic dilation of the left ventricle on stress thallium-201 scintigraphy: a marker of severe and extensive coronary artery disease. J Am Coll Cardiol 1987;9:752-9. 
Figure 1: Patient with a normal perfusion SPECT MPI on polar map (stress: upper panel; rest: lower panel) (A) and extensive calcifications in the calcium scoring scan of the left descending artery (LAD), left circumflex artery (LCX) and right coronary artery (RCA) with a total Agatston score of 1555 (B). The corresponding coronary angiography revealed multivessel disease with most severe lesions in the RCA (black arrows) and a total distal occlusion (white arrow) (C).

Figure 2: Distribution of patients $(n=50)$ with normal SPECT MPI and very high CAC $>1000$, stratified by the number of angiographically diseased vessels (0-, 1-, 2-, 3-VD).

Figure 3: Relationship between the number of diseased coronary vessels (0-, 1-, 2-, 3-VD) and calcium score (Agatston score). The circles demonstrate outliers. The Kruskal-Wallis $\mathrm{p}$-value for a difference of calcium scores between the groups amounts to 0.15 (n.s.). 


\section{TABLES}

Table 1 Baseline characteristics $(n=50)$

Male $36(72 \%)$

Age (y)

Mean \pm SD $\quad 67 \pm 9$

Range $\quad 51-87$

Framingham risk score $\quad 15 \pm 7.3$

Hypertension $\quad 42(84 \%)$

Dyslipidemia $\quad 24(48 \%)$

Diabetes $16(32 \%)$

Smoking $23(46 \%)$

Obesitiy $\left(>30 \mathrm{~kg} / \mathrm{m}^{2}\right) \quad 11(22 \%)$

Reasons for referral

Typical angina $\quad 14(28 \%)$

Atypical chest pain $\quad 6(12 \%)$

New onset of LBBB $1(2 \%)$

Syncope $1(2 \%)$

Preoperative evaluation $\quad 19(38 \%)$

Dyspnea $5(10 \%)$

Positive exercise test $\quad 4(8 \%)$

LBBB $=$ left bundle branch block 
Table 2 Angiographic CAD and revascularization

\begin{tabular}{llll}
\hline Angiographic CAD & Patients & $\mathrm{PCl}$ & CABG \\
0-VD & 13 & 0 & 0 \\
1-VD & 15 & 6 & 1 \\
2-VD & 10 & 6 & 1 \\
3-VD & 12 & 7 & 5 \\
total & 50 & 19 & 7 \\
\hline
\end{tabular}

CAD $=$ coronary artery disease

$\mathrm{VD}=$ vessel disease

$\mathrm{PCI}=$ percutaneous coronary intervention

$\mathrm{CABG}=$ coronary artery bypass grafting 


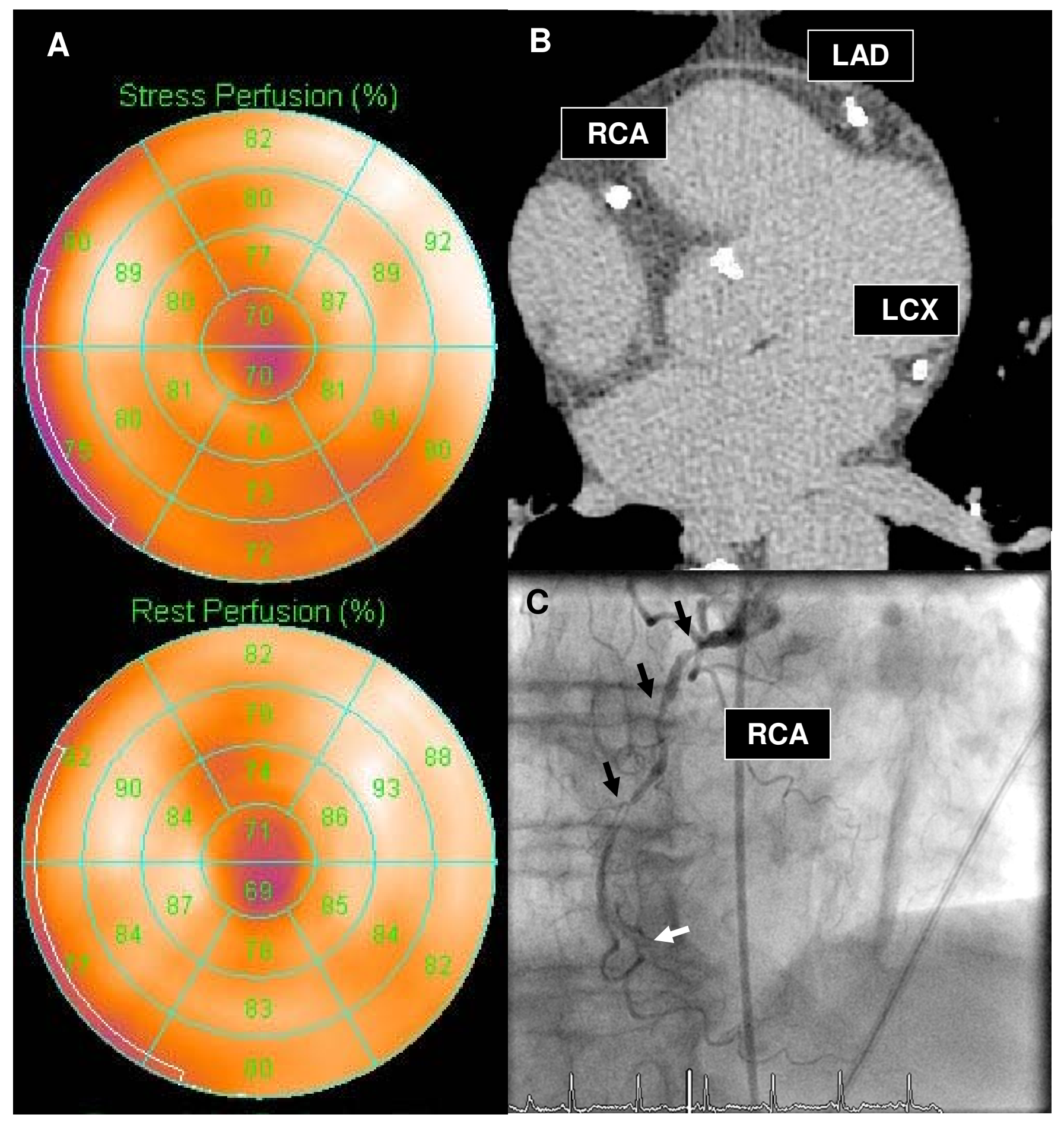

Figure 1 


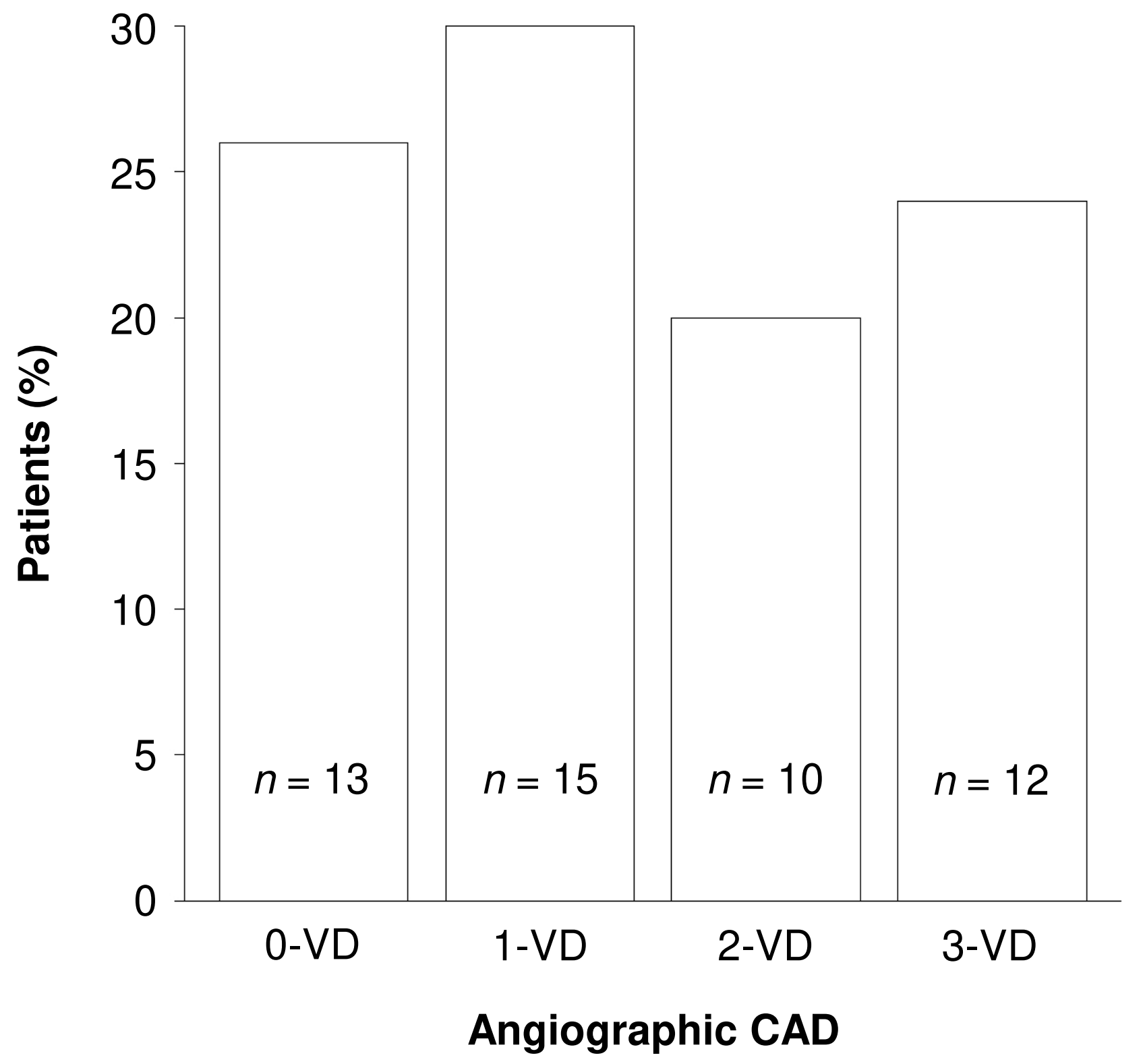

Figure 2 


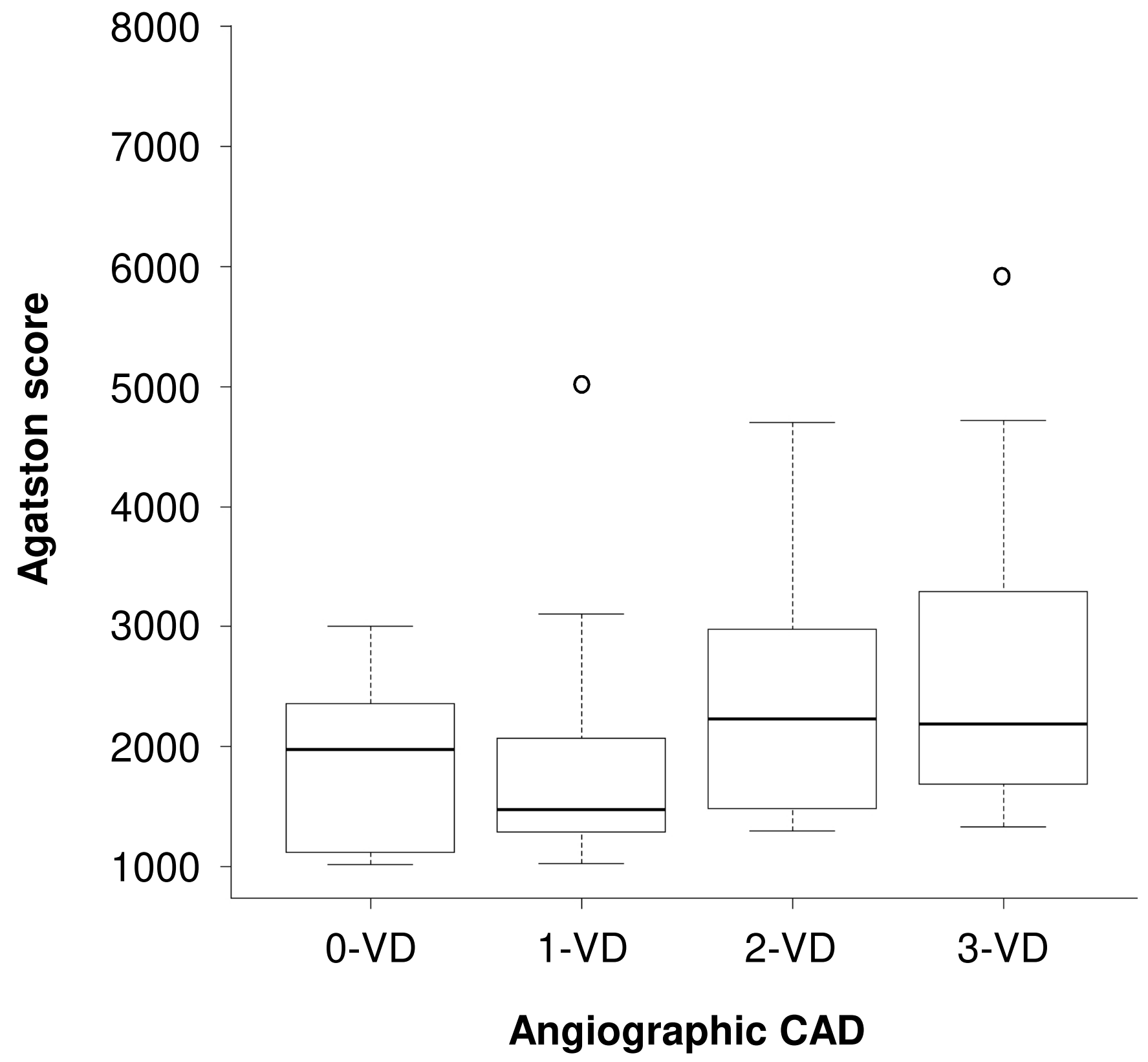

Figure 3 